\title{
Introduction to the special section on small bowel imaging
}

\author{
David H. Kim \\ University of Wisconsin, Madison, WI, USA
}

\section{Guest Editor}

Small bowel imaging has undergone a revolutionary change over the past decade. Advancements in hardware and software for both CT and MR now allow for exquisite evaluation of the small bowel with high spatial, contrast, and temporal resolution. Traditional small bowel fluoroscopic techniques have been largely replaced by cross-sectional counterparts. Although there is an unmistakable air of nostalgia with loss of these studies, the replacement modalities have led to an improved ability to detect small bowel abnormalities. Previously, this segment of the gastrointestinal tract routinely hid pathology. Now, even subtle bleeding vascular sources, small intraluminal masses, and mild inflammation can be seen. Work-up algorithms have been reorganized as imaging plays a much larger role in the evaluation of patients with inflammatory bowel disease, obscure GI bleeding, and other such indications.

It is our distinct privilege to present this special focus section in Abdominal Imaging on cutting-edge small bowel imaging. Leading imaging experts who comprise the Society of Abdominal Radiology (SAR) small bowel disease focus group provide excellent reviews on various small bowel topics including Crohn's disease and obscure gastrointestinal bleeding. Drs. Baker and Grand give insightful overviews of CT- and MR-based evaluation of Crohn's disease, respectively, including important technical and programmatic topics to maximize diagnostic accuracy. Dr. Anupindi extends the discussion to the pediatric population and Dr. Bruining, a gastroenterologist, nicely incorporates how imaging fits within the larger clinical perspective, with an emphasis on various imaging-based disease activity scoring indexes and likely uses in clinical trials. Finally, Dr. Soto provides an excellent discussion on the use of MDCT in the evaluation of obscure gastrointestinal bleeding. He nicely outlines the different uses of multi-phase angiographic CT and $\mathrm{CT}$ enterography, dependent on the specific bleeding scenario.

The editor's choice article for this month nicely reinforces the theme of advanced imaging improving identification of subtle small bowel pathology. NSAIDinduced small bowel strictures are an under-recognized disease entity, which have traditionally been difficult to visualize. Now with the advancements with $\mathrm{CT}$ and MR enterography, such lesions can be detected on a regular basis when present. In this editor choice article, Dr. Frye gives a comprehensive overview on NSAID strictures, including pathogenesis, imaging findings and pitfalls, endoscopic appearance, and treatment. As shown by this excellent article, NSAID-induced strictures have a characteristic presentation and appearance that can be suggested by the well-informed radiologist who is familiar with this condition.

Finally, an excellent collection of 12 additional articles rounds out this special feature section, including both original research and pictorial essays on various small bowel topics. These articles range from the use of advanced MR techniques in Crohn's disease management and dose reduction for CT enterography in Crohn's patients to performance of MDCT in closed-loop small bowel obstruction.

We are excited by the compilation of these excellent articles that comprise the special focus on small bowel in this issue. We believe that this informative issue should serve as a much referenced resource regarding state-ofthe-art small bowel imaging for the Abdominal Imaging readership in the coming years. 